\title{
Model infrared spectra of passively heated proto-planetary disks surrounding intermediate-mass pre-main-sequence stars
}

\author{
J. Meijer ${ }^{1}$, L. B. F. M. Waters ${ }^{1,2}$, A. de $\operatorname{Koter}^{1,3}$, M. Min $^{1}$, R. van Boekel ${ }^{4}$, C. Dominik ${ }^{1,5}$, and C. P. Dullemond ${ }^{4}$ \\ 1 Astronomical Institute, University of Amsterdam, Kruislaan 403, 1098 AJ Amsterdam, The Netherlands \\ e-mail: waters@uva.nl \\ 2 Instituut voor Sterrenkunde, Katholieke Universiteit Leuven, Celestijnenlaan 200D, 3001 Leuven, Belgium \\ 3 Astronomical Institute, Utrecht University, Princetonplein 5, 3584 CC Utrecht, The Netherlands \\ 4 Max Planck Institut Für Astronomie, Königstuhl 17, 69117 Heidelberg, Germany \\ 5 Department of Astrophysics/IMAPP, Radboud University Nijmegen, PO Box 9010, 6500 GL Nijmegen, The Netherlands
}

Received 8 February 2008 / Accepted 9 January 2009

\begin{abstract}
Aims. We study theoretical spectra at mid-infrared $(5-40 \mu \mathrm{m})$ wavelengths of proto-planetary disks surrounding intermediate-mass pre-main-sequence stars. Observations show a wide range of spectral shapes and a rich variety in strength and shape of dust resonances. These strong variations in spectral shape reflect differences in the nature and spatial distribution of dust particles in the disk. The aim of this study is to establish what model parameters influence the mid-IR spectra of planet-forming disks.

Methods. A grid of models of passively heated proto-planetary disks is used to calculate the infrared spectrum. We use hydrostatic equilibrium disk models and radiative transfer to calculate the emerging spectrum. We focus on the effects that different disk geometries (flaring, self-shadowed) and dust mineralogy have on the emerging 5-40 $\mu \mathrm{m}$ spectrum. We adopt four scenarios for the radial and vertical distribution of crystalline silicate dust.

Results. In our model, the $23.5 \mu \mathrm{m}$ forsterite band is more sensitive to emission from regions $<30 \mathrm{AU}$, while the $33.5 \mu \mathrm{m}$ forsterite band probes regions up to $50 \mathrm{AU}$. The $23.5 \mu \mathrm{m}$ band strength does not depend on the degree of flaring of the disk, while the $33.5 \mu \mathrm{m}$ band does. Only models with a substantial abundance ( $>5$ percent) of crystalline silicates at a long distance from the star $(>20-50$ AU) show detectable emission in the $33.5 \mu \mathrm{m}$ forsterite band. The carbon-dust abundance affects the strength of the dust resonances in the $10 \mu \mathrm{m}$ spectral region, but not in the $30 \mu \mathrm{m}$ region.
\end{abstract}

Key words. stars: planetary systems: protoplanetary disks - stars: pre-main sequence - stars: circumstellar matter

\section{Introduction}

Planet formation occurs in circumstellar disks that surround virtually all young low- and intermediate-mass stars. These disks are the remnant of the star formation process and are mostly heated by stellar photons (passive disks). They contain a modest amount of mass, usually 0.01 to $0.1 M_{\odot}$ (with a rather wide spread, see e.g. Hillenbrand et al. 1992; Henning et al. 1994). Initially, the disks are rich in gas and dust, but the gas and dust dissipate with time (typical timescales are $10^{6}$ to $10^{7}$ yr Haisch et al. 2001). A debris disk is left, which contains dust produced by the collisions of larger bodies in the young planetary system ( $\beta$ Pictoris is the most famous example).

The structure and evolution of proto-planetary disks are important pieces in the puzzle towards a better understanding of the way planetary systems form. The planet formation process is associated with large changes in the chemical composition of the gas and dust which enter the disk from the parent molecular cloud. In passive disks, the opacity of the dust is the most important parameter in determining the amount of stellar radiation which is reprocessed and thus the disk appearance (i.e. how strong the disk "flares"; D'Alessio et al. 1998; Dullemond \& Dominik 2004). The dust opacity in turn is strongly affected by grain aggregation, which removes small grains from the disk. Thus, grain aggregation and apparent disk structure are closely coupled. In addition, changes in the chemical composition of the dust may change its opacity, which also affects the apparent disk structure. In particular the presence of crystalline silicates may be used to study grain processing, since it is believed that such grains are produced in the disk, either by annealing of amorphous material close to the star, or by local heating processes at larger distance from the star. The spatial distribution of different grain materials may therefore be used to constrain physical and chemical processes that occur in proto-planetary disks.

Infrared spectroscopy of proto-planetary disks has proven to be a very powerful way to determine the nature of the warm grains in their surface layers (grain size, composition, and in particular crystallinity), and it also provides constraints on the global geometry of the disk (Meeus et al. 2001; van Boekel et al. 2005; Honda et al. 2004). Model calculations of infrared spectra of proto-planetary disks show that grain growth and settling strongly affect their spectral appearance (e.g. Dullemond \& Dominik 2004; D'Alessio et al. 2006). Mid-IR spectra in the 5-40 $\mu \mathrm{m}$ wavelength range have become available through the Infrared Space Observatory (ISO), and more recently, the Spitzer Space Observatory. The Spitzer archive by now contains hundreds of high quality disk spectra, showing a tremendous variation in spectral shape and presence of solid state bands, for a wide range of central star properties (e.g. Kessler-Silacci et al. 2006). The harvesting of this rich data set requires detailed modelling of emission from disks in hydrostatic equilibrium, including the effect of spatial variations in the dust properties on the emerging spectra. This type of models is urgently needed 
because the wide wavelength range of ISO and Spitzer allows probing a wide temperature range of the circumstellar dust, and so also a wide distance range. Models with a constant dust composition (both in grain size and crystallinity) throughout the disk are no longer capable of describing the detailed spectral structure seen in ISO and Spitzer data (e.g. Bouwman et al. 2001; Acke \& van den Ancker 2004; Kessler-Silacci et al. 2006), and are certainly not compatible with spatially resolved data (van Boekel et al. 2004).

This paper describes a study of model spectra of protoplanetary disks, using state of the art hydrostatic equilibrium models for passively heated proto-planetary disks (Dullemond \& Dominik 2004) surrounding intermediate-mass pre-mainsequence stars (Herbig Ae stars). In a previous study (Meijer et al. 2008), we have calculated a large grid of broad-band Spectral Energy Distributions (SEDs) to study the overall shape of the IR and millimetre spectrum of these models. In this paper we focus on the detailed spectral shape in the mid-IR, including resonances due to a number of abundant silicate dust species found to be present in proto-planetary disks. We focus on two aspects, namely disk geometry and radial distribution and size of different kinds of dust.

Disk geometry strongly affects the shape of the IR spectra. Observationally, "flaring" (group I) and "flat" (group II) disk geometries have been inferred from ISO observations of Herbig Ae/Be stars (Meeus et al. 2001). In their study of disk models surrounding Herbig Ae/Be stars, Dullemond \& Dominik (2004) showed that this empirical classification may correspond to flaring and self-shadowed disk geometries of passive disks in hydrostatic equilibrium, where the mass contained in small, micron or sub-micron sized dust grains determines whether a model is flaring (high dust mass) or self-shadowed (small dust mass). (To a lesser extent, also the surface mass density gradient determines disk geometry.) In this paper, we study the mid-IR spectra adopting two values for the mass contained in small dust grains, to simulate a group I and a group II spectrum. The total dust mass, i.e. the sum of small, sub-micron or micronsized dust and that of large, cold grains located in the disk midplane, is usually dominated by the mass in the mid-plane grains. This cold dust component is not considered further in this study, because it does not determine disk geometry and it also does not contribute significantly to the flux in the wavelength range of interest (Meijer et al. 2008). This implies that, when we talk about disk mass, we mean the mass of the small grains, scaled up by a canonical gas-to-dust ratio of 100 .

The second aspect we focus on in this paper is the spatial distribution of different dust species. We adopt several scenarios for the radial and vertical distribution of dust species such as crystalline silicates, and study the effect of these different spatial distributions on the $5-40 \mu \mathrm{m}$ wavelength range; this wavelength range contains a number of strong resonances from both amorphous and crystalline silicates. We focus on the effect of different dust compositions on the emerging spectrum, by adopting the disk structure from a self-consistent calculation using a standard (simplified) dust composition. This approach has the advantage that it allows us to determine the effect of changes in dust composition on the emerging spectra, knowing that the disk structure is the same.

This paper is organised as follows: in Sect. 2 we give an overview of the disk models used for this study and introduce the adopted descriptions for radial and vertical mixing. In Sect. 3 we present a parameter study of the mid-IR spectra. Section 4 discusses the behaviour of the 23.5 and $33.5 \mu \mathrm{m}$ forsterite band strengths as a function of specific model parameters. Section 5 presents the conclusions of this paper.

\section{The disk model}

We use the code described in Dullemond \& Dominik (2004) to create models for the spatial and temperature structure of protoplanetary disks. This code solves $2 \mathrm{D}$ radiative transfer subject to the constraints of radiative equilibrium and vertical hydrostatic equilibrium in an iterative way. We assume a passive disk and therefore do not take into account any heating by viscous accretion. Calvet et al. (2000) give a critical mass accretion rate of $2 \times$ $10^{-8} M_{\odot} \mathrm{yr}^{-1}$ for classical T Tauri stars below which irradiation dominates the inner disk heating. For a typical HAeBe star this becomes approximately $10^{-7} M_{\odot} \mathrm{yr}^{-1}$. Since intermediate-mass stars that are close to or on the main sequence typically have accretion rates of $10^{-8} M_{\odot} \mathrm{yr}^{-1}$ or less (Calvet et al. 2004; Natta et al. 2006) the assumption of a passive disk is justified. The model describes a disk only, i.e. we do not assume the system to be embedded in an extended (spherical) halo. Effects of scattered radiation are not taken into account. According to Dullemond \& Natta (2003) this may introduce an overestimate of the $1-10 \mu \mathrm{m}$ flux of up to a few tens of percent.

For a more detailed description of the adopted set of $2 \mathrm{D}$ radiative transfer models of disks in vertical hydrostatic equilibrium we refer to Meijer et al. (2008, hereafter referred to as Paper I), where the behavior of the spectral energy distribution is compared to observations.

\subsection{Basic disk parameters and dust mineralogy}

From a study of the morphology of the spectral energy distribution (SED) Meeus et al. (2001) classified Herbig stars in two groups, labeled Group I and Group II, reflecting an "outward flaring" and a relatively "flat" geometry, respectively (Dullemond \& Dominik 2004). Disks flare if the opaqueness of the grains, to which small particles contribute the most, is high. To further clarify this important point: the difference between a flaring and a non-flaring disk need not reflect distinctly different vertical density structures of the gas and dust. The vertical structures, set by hydrostatic equilibrium, will actually be very similar for a wide range in grain properties as the temperatures in the mid-plane layers (setting the structure through the vertical scale-height) are rather insensitive to these properties (see also Dullemond et al. 2007).

Nevertheless, to account for small differences in the vertical disk structure resulting from the differences in dust opaqueness, the Group I sources are represented by a disk with a total mass of $0.05 M_{\odot}$ and Group II sources by a disk of $10^{-4} M_{\odot}$. We note that gas is assumed to contribute 100 times as much to this total mass as does the dust. The surface density structure $\Sigma(r)$ of the disks is described by a radial power law. We adopt a value of -1.5 for this powerlaw, corresponding to that used in the minimum mass solar nebula model (Weidenschilling 1977). A recent discussion concerning the mass surface density gradient in proto-planetary disks can be found in Desch (2007).

To compute the vertical structure we assume "astronomical silicate" (Laor \& Draine 1993) and carbon (Preibisch et al. 1993) particles. We used two sets of carbon abundances, leading to a carbon poor disk structure and a carbon rich disk structure. The carbon poor disk structure consists of "astronomical silicate" only. The carbon rich disk structure consists of a mixture of 5/6 "astronomical silicate" and 1/6 carbon (by mass). We note 
that the "astronomical silicate" opacities are empirically derived, therefore the precise chemical composition responsible for these opacities is not well known. In fact they may contain some contribution from carbon, since they were derived from interstellar dust absorption lines of sight (Draine \& Lee 1984).

Although these different dust compositions have only a very minor effect on the resulting vertical disk structure, we decided to use the disk structure of the carbon-poor and carbon-rich models that most closely matched the carbon abundance of the detailed dust composition models (see below) used to calculate the emerging spectra. We assume that the dust grains are thermally coupled, i.e. all grain species have the same temperature where they co-exist.

The size distribution of grains is assumed to follow a powerlaw, with index -3.5 , and to range from a minimum size of $0.01 \mu \mathrm{m}$ to a maximum size of $1 \mu \mathrm{m}$. This is consistent with collisional fragmentation considerations (Hellyer 1970) and is similar to the size distribution of interstellar grains (Mathis et al. 1977). The optical properties of the grain population were combined into a single grain to ease computation. Grains are homogeneously distributed throughout the disk, i.e. we do not consider a (relatively cold) mid-plane layer of very large grains as for instance adopted by Dullemond \& Dominik (2004). Omitting this cold mid-plane layer of dust does not affect the spectral shape in the wavelength range we discuss here $(5-40 \mu \mathrm{m})$, since the mid-plane only begins to contribute at longer wavelengths.

In predicting the spectral signature in detail we adopt the disk density structure as it follows from this simple description of grain opacities, but recalculate the grain temperatures and emergent spectrum introducing a more detailed dust description (see below). The opacities of this more detailed dust description may affect the vertical opacity structure, therefore (to some extent) the amount of disk flaring.

The inner radius of the dust disk is computed selfconsistently. This is done by assuming the temperature at the inner radius to be the sublimation temperature, for which we adopt $T_{\text {sub }}=1500 \mathrm{~K}$, and then calculating the inner radius using the assumption of a vertical wall (for details see Dullemond \& Dominik 2004). Although a rounded off inner rim such as described by Isella \& Natta (2005) is physically more desirable, we will use a vertical inner rim for ease of calculation. The outer radius of our models is set to $200 \mathrm{AU}$. Beyond this radius the surface density falls of with a power of -12 to prevent the occurrence of too abrupt changes in optical depth. The density profile perpendicular to the disk mid-plane is determined from vertical hydrostatic equilibrium.

The sample of Herbig stars to which we will compare our calculations show little or no optical extinction. (see van Boekel et al. 2005). This implies that we do not view these systems at very high inclination angles as otherwise significant optical extinction of the stellar spectrum by the disk must occur. In our model calculations we assume to view the disk at an inclination angle of 6 degrees, i.e. almost pole-on. Using a pole-on inclination would introduce modelling artifacts due to the perfectly vertical inner rim.

The central star has a mass of $2.5 M_{\odot}$, an effective temperature $T_{\text {eff }}=10000 \mathrm{~K}$, and a luminosity that is 40 times that of the sun. These values are typical for Herbig Ae stars.

The behaviour of the spectral energy distribution of the models selected for this study are compared to observations in Meijer et al. (2008), to which we refer for a further discussion. Here we suffice by stating that they are in fair agreement with global properties of observed SEDs.

\subsection{Grain composition and properties used to compute model spectra}

The disk models described above are used to calculate infrared spectra in the 5-40 $\mu \mathrm{m}$ wavelength range. We know from many observations that the actual dust composition that can be inferred from the spectra is far more complicated than the one used above to compute the disk structure. One of the main goals of this paper is to study the effect of spatially varying dust composition on the resulting model spectra. These spatial variations in dust composition result from annealing of amorphous dust and other processes (see below). We therefore introduce new dust species for the calculation of the model spectra; in particular we need to consider both amorphous and crystalline particles. For the opacity of the crystalline material we use a mixture of $50 \%$ forsterite (Servoin \& Piriou 1973) and 50\% enstatite (Jäger et al. 1998). Gail (2004) shows that inward of the radius ( $\sim 0.6$ AU) where diffusion to the outer disk becomes important there should be an equal amount of forsterite and enstatite present. Although the forsterite is expected to be more concentrated to the innermost part of the disk, we will treat the different crystalline silicates as one crystalline component. For the opacity of the amorphous grains we use olivine $\left(\mathrm{Mg}_{2 x} \mathrm{Fe}_{2-2 x} \mathrm{SiO}_{4}\right)$. We use three values for the carbon dust abundance, $5 \%, 15 \%$ and $30 \%$. The $5 \%$ model spectra are calculated using the model structure without any carbon included (see above), while for the $15 \%$ and $30 \%$ carbon dust abundance models we use the carbon-rich model structure. Note that there are only minor differences between the carbonpoor and carbon rich disk models.

The dust opacities are calculated from laboratory measurements of the refractive index as a function of wavelength, following Min et al. (2005). Observations of interplanetary dust particles (Warren et al. 1994) and fitting of spectra using spherical homogeneous grains (see e.g Hony et al. 2002; Bouwman et al. 2001) strongly indicate that astronomical dust grains may be non-spherical. Since modelling of the exact shapes of dust grains is not yet feasible Min et al. (2005) take a statistical approach (suggested by Bohren \& Huffmann 1983) in accounting for deviations of perfect spherical particles. In this approach the optical properties of an ensemble of irregularly shaped dust grains are simulated by the optical properties of an ensemble of particles with simple shapes. As Min et al. (2003) show that there is no significant difference between different non-symmetrical shapes when using the statistical approach, they have chosen the one that is easiest to compute, i.e. a hollow sphere. This shape has the advantage of having optical properties that are easy to compute for almost all wavelengths and grain sizes. We assume an ensemble of such grain shapes in computing the opacity of the crystalline silicates. For the amorphous grains we suffice with homogeneous spheres, as for these particles the effect of nonsphericity is modest.

In order to account for different sizes of the dust particles, we use combinations of two different sized grains: small $(0.1 \mu \mathrm{m})$ and large $(1.5 \mu \mathrm{m})$. Bouwman et al. (2001) showed that the emission of dust grains contributing to the $10 \mu \mathrm{m}$ silicate feature can be well characterised by taking into account only two typical sizes: $0.1 \mu \mathrm{m}$ for grains with sizes smaller than $1 \mu \mathrm{m}$, and $2 \mu \mathrm{m}$ for grains with sizes larger than $1 \mu \mathrm{m}$. This approach was followed by Honda et al. (2004), though van Boekel et al. (2005) found that using $2 \mu \mathrm{m}$ for the large grains would overestimate the abundance of large crystalline grains and hence the crystallinity. Therefore we adopt their large grain size of $1.5 \mu \mathrm{m}$. We adopt three grain size mixtures in our models: small grains only, an 

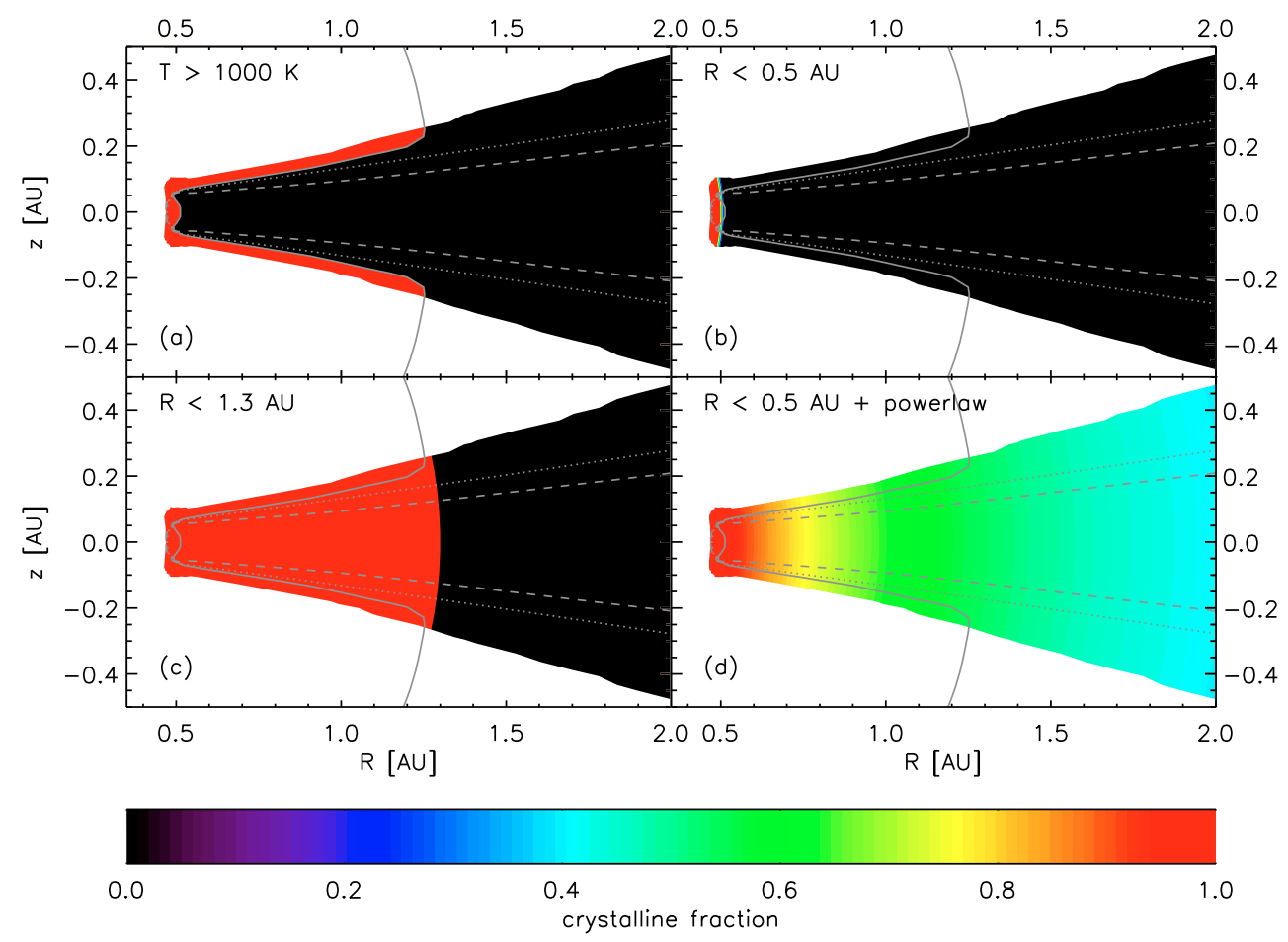

Fig. 1. The geometry of the inner disk for each of the four scenarios, which have crystalline grains at A: $T>1000 \mathrm{~K}$; B: $R<0.5$ AU; C: $R<1.3 \mathrm{AU} ; \mathrm{D}: R<0.5 \mathrm{AU}+$ power law. All models have only small grains and contain 30 percent carbon by mass. The colour scale shows the fraction of crystalline material. The solid line is the $1000 \mathrm{~K}$ contour. The dotted line gives the locus where the optical depth in the visual along radial rays, $\tau_{\mathrm{V}}$, reaches unity. The dashed line shows where the optical depth at $10 \mu \mathrm{m}$ in the vertical direction, $\tau_{10 \mu \mathrm{m}}$, is unity.

equal amount of small and large grains; and 90 per cent large grains.

\subsection{Simple descriptions of radial and vertical mixing}

To investigate the effects of mixing of crystalline grains throughout the disk, we have devised four simple models describing the radial and vertical mixing of material. The distributions of crystalline grains in these models are shown in Fig. 1. Before explaining the four scenario's in more detail, let us first explain the meaning of the grey-scale and contours. The grey-scale shows the fraction of crystalline material quantified by the horizontal bar below the figure. The dotted line shows the surface for which the optical depth in the visual $\tau_{\mathrm{V}}$ along radial rays equals unity. This is roughly the region where the disk absorbs the stellar light and where the temperature shows a steep gradient. The disk can be split along this $\tau_{\mathrm{V}}=1$ surface into a hot "chromospheric" layer (above the $\tau_{\mathrm{V}}=1$ surface) and a cooler interior. The solid line shows the $1000 \mathrm{~K}$ contour. It follows the surface layer until the point where the highest temperature that is reached in the chromospheric zone drops below this value, where it curves upwards. As no halo is accounted for, the medium high above the disk surface contains very little material (a minimum density of $10^{-95} \mathrm{~g} \mathrm{~cm}^{-3}$ is forced) and does not contribute to the spectral properties.

The dashed line shows the locus for which the optical depth at $10 \mu \mathrm{m}$ in the vertical direction $\tau_{10 \mu \mathrm{m}}$ equals unity. As the temperature stratification in the surface layers is such that the Eddington-Barbier relation does not apply (as in this regime the temperature gradient in the vertical direction is not represented well by a linear function in optical depth) the $\tau_{10 \mu \mathrm{m}}=$ 1 surface cannot be identified with the location from where the emergent specific intensity (appears) to originate. At most it gives an indication of what layers of the disk surface contribute to the SED at this wavelength.

One of the central questions in the evolution of protoplanetary disks is the importance of radial mixing in their active, and possibly even passive phase (e.g. Bockelée-Morvan et al. 2002; Gail 2004; Dullemond et al. 2006). Crystalline silicates may be used as a probe of such processes because the thermal history of these grains is imprinted in their lattice structure. Two processes can cause grains to be crystalline: (1) gas-phase condensation at equilibrium temperature and pressure, important in the innermost hottest regions of the disk; and (2) annealing of amorphous grains at slightly lower temperature, but above the annealing temperature of silicates, which is estimated to be about $1000 \mathrm{~K}$. Both processes are likely important in the inner disk regions and would therefore naturally result in a high inner disk crystallinity. Radial mixing in the disk may transport significant amounts of this material to the outer disk regions, but at present it is uncertain how efficient this mechanism is. There are other annealing processes that may occur far from the star, thus also increasing crystallinity at large distance (without radial mixing). These local processes include lightning and shocks (spiral arms due to planet formation).

We have defined four scenarios to investigate the effect of different spatial distributions of crystalline dust on the mid-IR spectra of proto-planetary disks. These scenarios are intended to probe (the extremes of) parameter space. We adopt a temperature of $1000 \mathrm{~K}$ as the lower limit for grains to anneal. This may be pessimistic, i.e. grains may anneal at lower temperature depending on chemical composition, grain size and other parameters. In that case our calculations are conservative and give a 
Table 1. Model parameters.

\begin{tabular}{lrr}
\hline \hline Parameter & Standard value & Range \\
\hline$R_{\star}$ & $2.1 R_{\odot}$ & \\
$M_{\star}$ & $2.5 M_{\odot}$ & \\
$T_{\text {eff }}$ & $10000 \mathrm{~K}$ & \\
$L_{\star}$ & $40 L_{\odot}$ & \\
Distance & $150 \mathrm{pc}$ & \\
\hline \multicolumn{4}{c}{} & \\
\hline Disk mass & disk & $5 \%, 15 \%, 30 \%$ \\
Disk inclination angle & 0.1 rad $\left(\approx 6^{\circ}\right)$ & $0 \%, 50 \%, 90 \%$ \\
Surface mass density gradient & -1.5 & \\
Gas to dust ratio & 100 & \\
Carbon abundance & $15 \%$ & \\
ratio large/small grains & $50 \%$ & \\
Crystallinity scenario & D, with $p=-0.65$ & $-0.30,-0.65,-1.00,-1.50$ \\
\hline
\end{tabular}

minimum amount of crystals due to inner disk annealing. The four scenarios, $(A)$ through $(D)$, are defined as follows:

A) No radial mixing: the dust is crystalline where it reaches a temperature above $1000 \mathrm{~K}$. Crystalline silicates are located in the inner disk region. Two regions of the inner disk contain crystalline silicates: (i) the inner disk is completely crystalline up to a distance of about $0.5 \mathrm{AU}$; (ii) a thin layer in the upper disk atmosphere is crystalline up to a distance of about 1.3 AU. This scenario has no vertical mixing.

B) The minimum crystallinity scenario: assumes that the disk is fully crystalline only in the region where the mid-plane temperature is above $1000 \mathrm{~K}$. This implies ignoring the chromospheric layer on the disk that was also crystalline in scenario A. Scenario B has the minimum crystallinity one can reasonably imagine. This scenario could in principle be interpreted as short-duration, fast vertical mixing. The mixing would offset the ongoing crystallisation in the chromospheric layer by bringing up amorphous material from the mid-plane in that region. This scenario is not physically realistic as the timescale of crystallisation in the chromospheric layer is extremely short (at $1000 \mathrm{~K}$ particles are annealed in minutes) compared to the timescale associated with vertical mixing.

C) The vertical mixing scenario: this scenario implies efficient vertical mixing, continuing for a long time. The temperature of the chromospheric layer exceeds $1000 \mathrm{~K}$ out to a distance of about 1.3 AU. We assume now that vertical mixing has transported crystalline material down so that the entire vertical slice ending in the $>1000 \mathrm{~K}$ chromosphere becomes fully crystalline.

D) The radial mixing scenario: in this scenario, we assume a radial profile of the crystallinity. We start from scenario B with $100 \%$ crystallinity at the inner rim. We then introduce a power-law dependence of the crystallinity with distance from the star. The default value for the power-law index is -0.65 , as derived from detailed mixing/annealing models (Gail 2001). We always take the crystallinity to be constant in each vertical slice, and we assume crystalline and amorphous components to be in thermal contact.

While for simplicity we call this case the "radial mixing scenario", it can also be interpreted as the result of distributed local crystallisation processes like shocks or lightning.

It is useful to also investigate the effect of different radial mixing efficiencies in the context of scenario D. We therefore also computed several models with other radial gradients of the crystallinity. We plot the crystallinity as a function of distance for several choices of the radial gradient in scenario D models in Fig. 9.

\subsection{Summary of model parameters}

We define two "standard models" with model parameters as summarised in Table 1, whose properties we will first study, and that will be used as a reference for the description of the parameter study. We use the following code to refer to our grid of models: $\{\mathrm{ABCD}\}\{1,2\}\{\mathrm{Lxx}\}\{\mathrm{Cxx}\}\{\mathrm{Pxxx}\}$, referring to $\{$ scenario $\}\{$ group $\}$ fraction of large grains $\}\{$ carbon abundance $\}$ sscenario D crystallinity gradient, if applicable\}. In this notation the standard models we have defined are D1L50C15P065 and D2L50C15P065, respectively. This means that the standard model has 50 percent large grains, has a crystallinity gradient $p=-0.65$ and a carbon abundance of 15 percent. Table 1 gives the range of model parameters used in our parameter study.

In Fig. 2 we show, for the wavelength range of the model spectra discussed in this paper, the dust opacities of the three components (amorphous silicate, crystalline silicate, and carbon) used in the standard model, i.e. for the grain size mixture containing 50 percent large grains.

\section{Description of the model spectra}

We now discuss the emerging spectra in the wavelength range 7-40 $\mu \mathrm{m}$, which covers most of the strongest resonances of abundant silicates, both crystalline and amorphous. The chosen wavelength range also covers that of the Spitzer IRS (Houck et al. 2004) and of the ISO Short Wavelength Spectrograph (SWS) (de Graauw et al. 1996), allowing detailed comparisons between models and observations. We first discuss changes in the radial distribution of the crystalline silicates, followed by changes in the grain size and the abundance of carbon dust.

\subsection{Changing the radial distribution of the crystalline silicates}

Figure 3 shows the model spectra for our standard model group I and group II sources (scenario D, bottom panels), and compares these model spectra to those calculated for the scenarios A through $\mathrm{C}$, adopting the standard values for carbon abundance and grain size. The overall shape of the standard group I and 


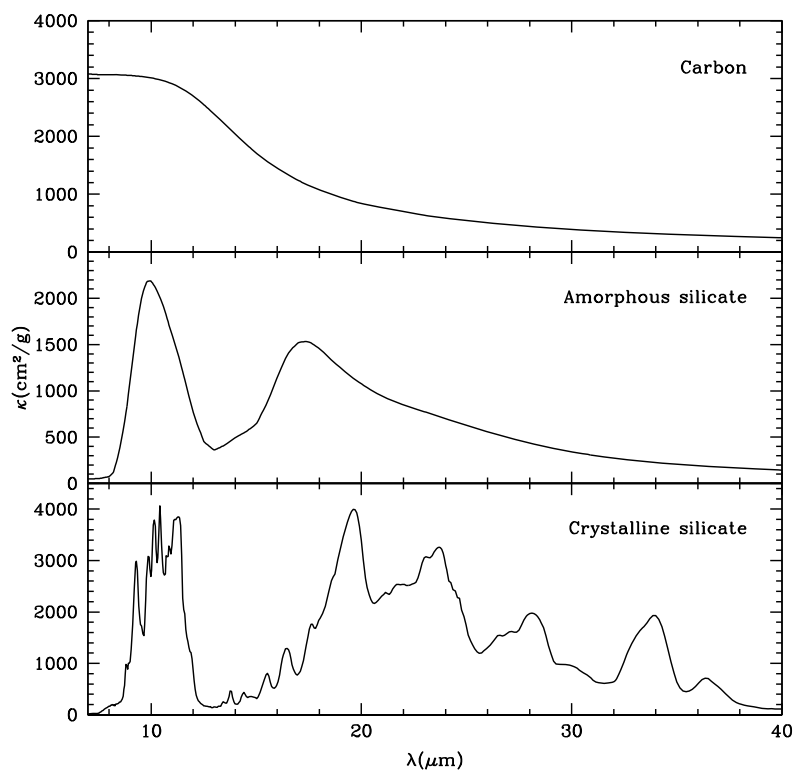

Fig. 2. The opacities of the used dust species used to calculate the model mid-IR spectra for the standard group I and group II models. These opacities are based on a grain size mixture containing equal amounts by mass of small $(0.1 \mu \mathrm{m})$ and large $(1.5 \mu \mathrm{m})$ grains.

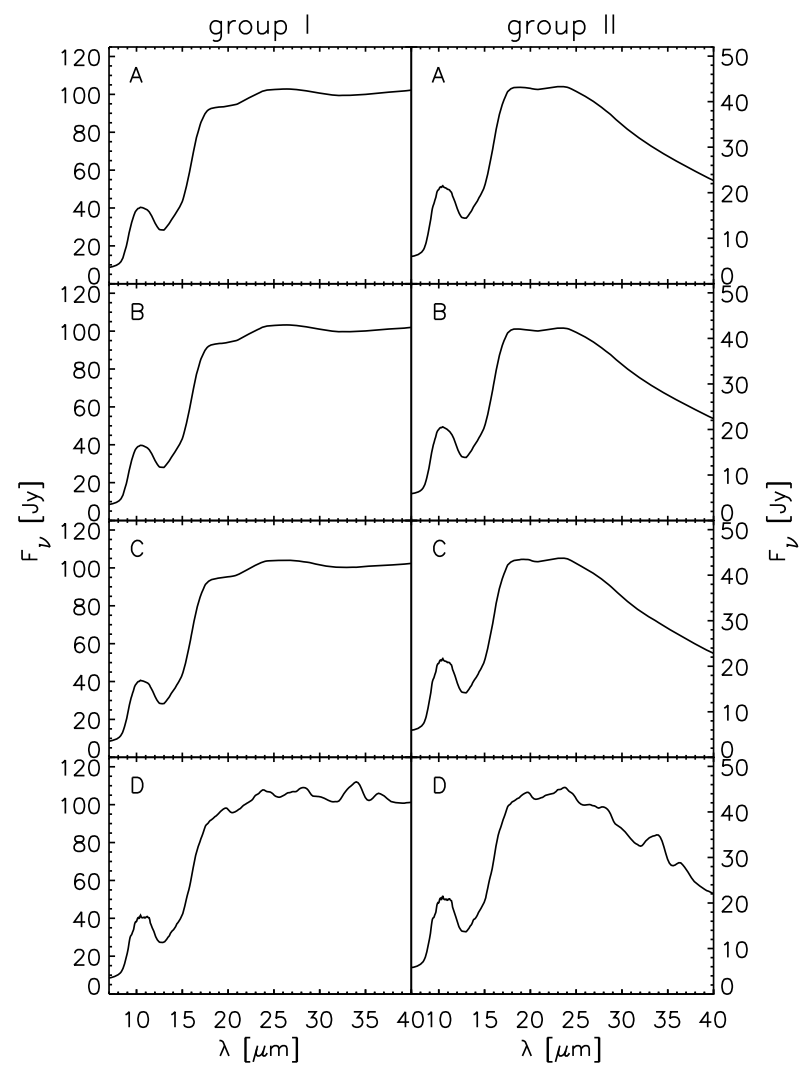

Fig. 3. The mid-IR spectra of the group I and group II models are shown for the four different mixing scenarios. Note that in scenarios D (bottom panels) the crystalline silicate features are prominent.

group II model spectra is characterised by a rising continuum between 7 and about $20 \mu \mathrm{m}$, followed by a decrease longward of $20 \mu \mathrm{m}$ for the group II model. The group I model has a roughly flat spectrum between 20 and $40 \mu \mathrm{m}$. This difference in spectral shape can be attributed to differences in the degree of flaring of group I (large flaring) and group II (little flaring) (see also
Dullemond \& Dominik 2004; Meijer et al. 2008). Interestingly, the rise in flux between 10 and $20 \mu \mathrm{m}$ is similar in both disk models, with the group I models having a somewhat steeper slope than the group II models. Clearly, the distinction between group I and group II is most evident at longer wavelengths, where the cool dust present in the flaring part of group I disks contributes significantly to the total flux. The flux levels of group I models are a factor 2 to 3 higher than those of group II models. This reflects the difference in solid angle the disks subtend when viewed from the star.

Both spectra for scenario D show emission bands from amorphous $(9.7$ and $18 \mu \mathrm{m}$ ) and crystalline (mostly the 11.3, 19.5, 23.5, 27.5, and $33.5 \mu \mathrm{m}$ bands of forsterite, $\mathrm{Mg}_{2} \mathrm{SiO}_{4}$ ) silicates. These bands are formed in the upper layers of the disk, where the temperature is (much) higher than in the mid plane. The signature of crystalline silicates is prominent in both spectra, indicating that for our standard model the abundance of crystalline silicates is high enough to produce a detectable signature even for the $33.5 \mu \mathrm{m}$ band, which probes the coolest crystalline dust in our spectra.

The other six panels in Fig. 3 show the model spectra for scenarios A-C. Their overall spectral shape is similar to that of the standard model D spectra. This can be understood by noting that the models have all been derived from very similar density structures (see Sect. 2). Interestingly, the signature of crystalline silicates in the scenario A-C spectra is very weak. Only near $10 \mu \mathrm{m}$ the shape of the amorphous silicate band is somewhat affected, and a shoulder near $11.3 \mu \mathrm{m}$ becomes visible for scenario $\mathrm{C}$. We conclude that substantial amounts of crystalline silicate dust must be present far from the star for mid-IR spectra to show emission from crystalline silicates.

In a separate paper (Meijer et al. 2007) we discuss in some detail the $10 \mu \mathrm{m}$ silicate band calculated from our models, and we will compare these model calculations to observations.

The effect of changing the radial mixing gradient (parameter $p$ ) in scenario D is shown in Fig. 4. The spectra show a dramatic increase in crystallinity going from $p=-1.50$ to -0.30 . Group II models tend to show somewhat stronger crystalline silicate bands compared to group I models, due to the fact that in group II models the flux is more dominated by the inner disk regions. Note also that changing the radial density gradient has much more effect on the longer wavelength spectra than on the shape of the $10 \mu \mathrm{m}$ spectrum. For instance, compare the P030 and P065 models. The $10 \mu \mathrm{m}$ silicate band probes the inner disk regions. These inner regions are highly crystalline for both values of $p$, and so the band shape is similar (but not equal) for both models. The $33.5 \mu \mathrm{m}$ band is sensitive to the degree of crystallinity in the outer disk regions, where the crystallinity is much different, resulting in strongly differing $33.5 \mu \mathrm{m}$ band strengths.

\subsection{Changing the carbon abundance}

In Fig. 5 we show the effect of changing the carbon dust abundance on the resulting spectra. We use the standard model assumptions for all other model parameters (see above). The overall spectral shape of the models is unaffected. However the band strength (band over continuum ratio) of the $10 \mu \mathrm{m}$ amorphous silicate band decreases significantly with increasing carbon dust abundance. This is much less the case for the longer wavelength crystalline silicate bands. The reason for this difference is primarily due to differences in the wavelength dependence of the silicate and carbon opacities. This is evident from Fig. 2, and can even more clearly be seen in Fig. 6. This latter figure shows the wavelength dependence of the dust opacity using a mixture of 


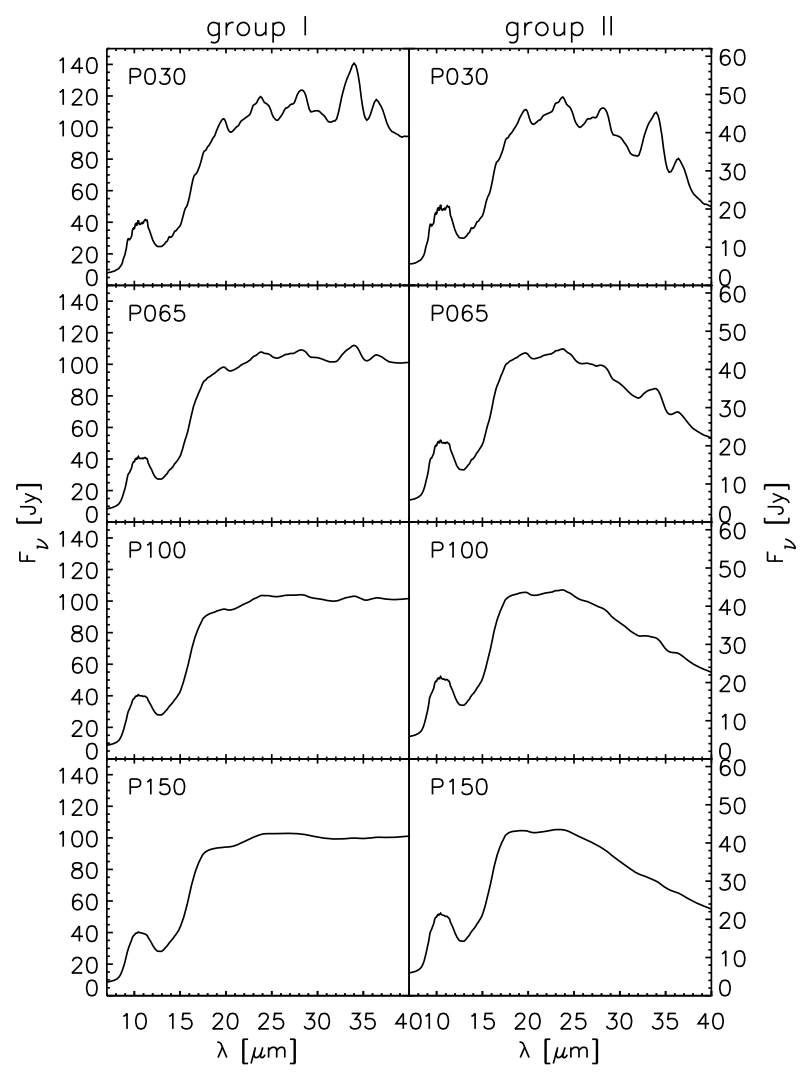

Fig. 4. The mid-IR spectra of the group I and group II models are shown for scenario $\mathrm{D}$ with different choices of the radial gradient of the crystallinity (parameter $p$ ): $p=-1.50,-1.00,-0.65$ and -0.30 from bottom to top. Note that with increasing value of $p$ the spectra become much smoother, due to a rapid decrease in the amount of crystals far from the star.

silicates (90 percent amorphous, 10 percent crystalline) and carbon dust $(5,15$ or 30 percent of carbon with respect to silicate). We use a grain size consisting of 50 percent large and 50 percent small grains. The strength of the $10 \mu \mathrm{m}$ silicate resonance as measured against the adjacent "continuum" opacity decreases significantly as the carbon dust opacity increases. However, the strength of the $33.5 \mu \mathrm{m}$ forsterite band measured against its adjacent "continuum" is hardly affected. This implies that the derivation of the amount of crystalline material from the $33.5 \mu \mathrm{m}$ band strength does not depend on the adopted carbon dust abundance. The derivation of the abundance of small silicate particles (amorphous and crystalline) from the $10 \mu \mathrm{m}$ spectral region does depend on the used carbon dust abundance. This issue will be further discussed in Meijer et al. (2007). We stress that both spectral regions also probe very different spatial regions in the disk!

\subsection{Changing the large grain abundance}

Finally, in Fig. 7 we study the effect of changing the grain size on the model spectra. The most striking changes are in the overall shape of the spectra: flux levels in the $15-40 \mu \mathrm{m}$ wavelength range decrease by 20 percent (group I) to 70 percent (group II) as the large grain fraction increases from 0 to 90 percent. The ratio of IR to stellar luminosity decreases from 0.54 to 0.49 for the group I models, and from 0.24 to 0.19 for group II models. This is due to the fact that with increasing large grain fraction the opacity of the dust at short wavelengths strongly decreases, resulting in a smaller disk opening angle within which stellar

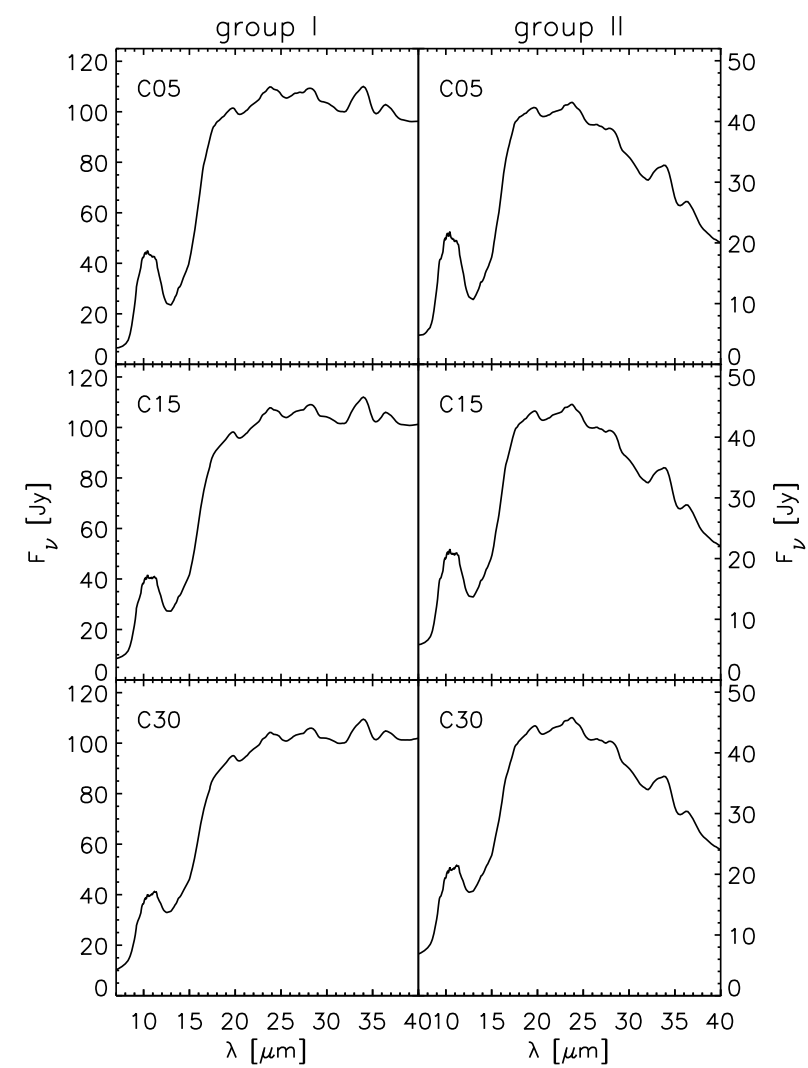

Fig. 5. The mid-IR spectra of the group I and group II models are shown for the standard scenario D (middle panel in each column), as well as for two additional models in which the carbon dust abundance is changed to 5 percent (top panels) and 30 percent (lower panels).

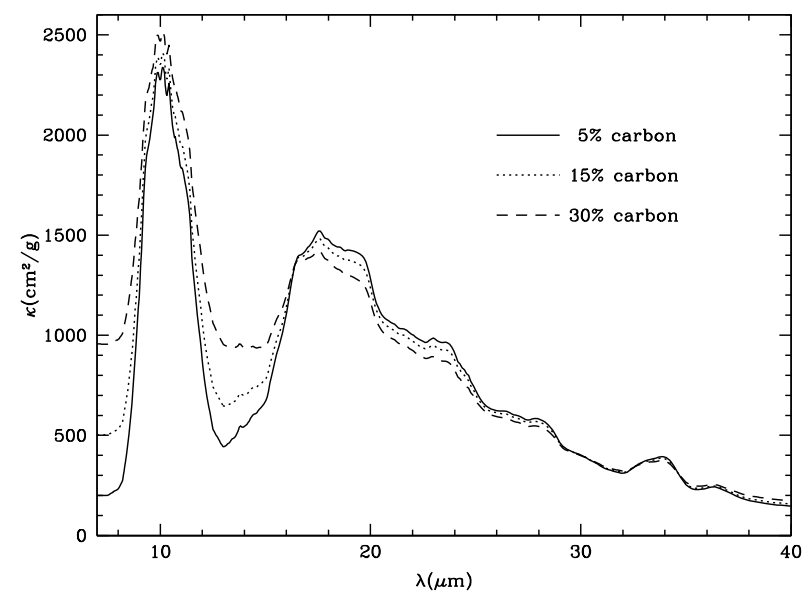

Fig. 6. Opacity of a mixture of carbon dust and silicate dust. The silicate dust consists of 90 percent amorphous silicate and 10 percent crystalline dust (equally divided between forsterite and enstatite). Three values of the carbon dust opacity are used, namely 5,15 and 30 percent. A mixture of 50 percent large and 50 percent small grains was adopted. Notice the different importance of the carbon dust opacities in the $10 \mu \mathrm{m}$ and the $30 \mu \mathrm{m}$ spectral region.

radiation is efficiently absorbed by the star. The band shape of the $10 \mu \mathrm{m}$ silicate band is also affected, since the strong resonances of amorphous and crystalline silicates in this wavelength region are very sensitive to grain size changes as used in this analysis. The longer wavelength crystalline silicate band opacities are much less sensitive to the grain size changes considered here. 


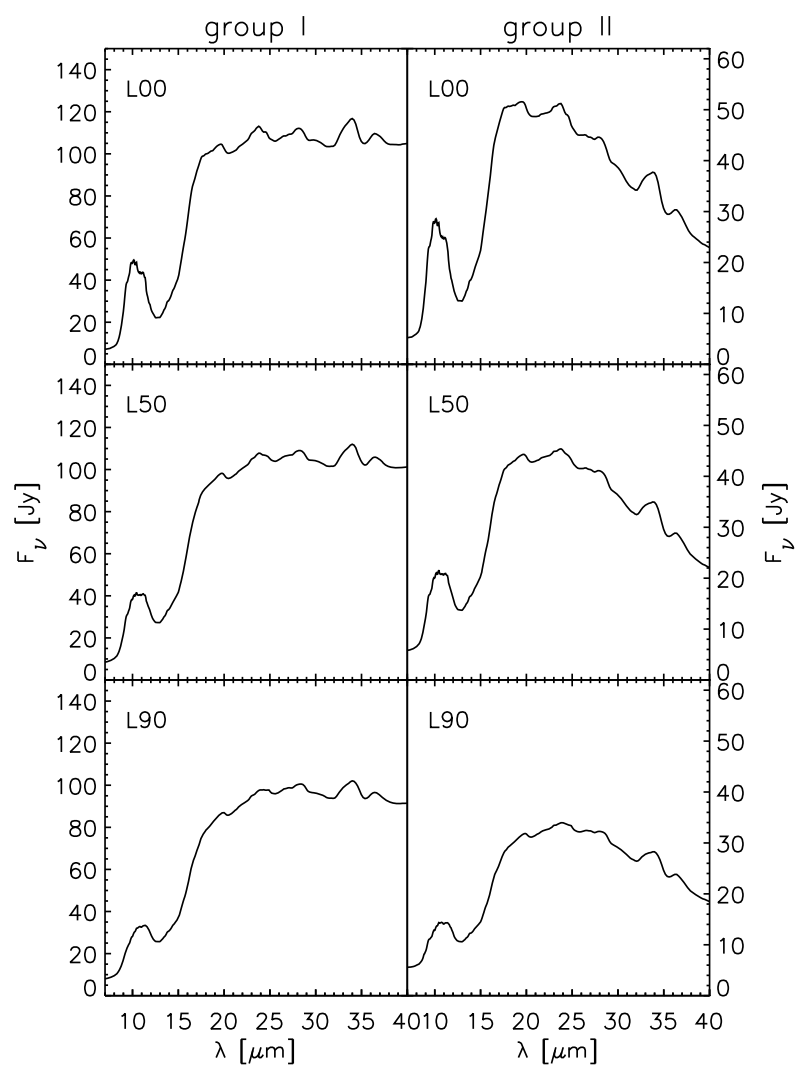

Fig. 7. The mid-IR spectra of the group I and group II models are shown for the standard scenario D (middle two panels in each row), as well as for two additional models in which the grain size is changed to small grains only (top panels) and 90 percent large grains (lower panels). We recall that with small grains we mean $0.1 \mu \mathrm{m}$ and large $1.5 \mu \mathrm{m}$.

\section{Forsterite band strengths from our model spectra}

In the previous section we have shown the sensitivity of model spectra to selected model parameters. From an observational point of view, it would be very useful if simple observables such as the band strength of crystalline and amorphous silicates could be used to distinguish between scenarios. An obvious question is in what manner the strength of these bands depend on disk geometry and thus disk mass. To investigate this further, we calculated several scenario D models with dust masses in between those used to generate the standard group I and group II models. The 23.5 and $33.5 \mu \mathrm{m}$ forsterite bands are prominent in the model spectra for models with small $p$, i.e. $p=-0.30$ and $p=-0.65$ (see Fig. 4) and we focus our discussion on these two values of $p$. The results are shown in Fig. 8.

Both the 23.5 and $33.5 \mu \mathrm{m}$ bands are sensitive to the value of $p$, and are stronger for $p=-0.30$. This can be easily understood by noting that the amount of crystalline material is higher in the $p=-0.30$ model (see Fig. 9). The $33.5 \mu \mathrm{m}$ band however is more sensitive to changes in $p$ than the $23.5 \mu \mathrm{m}$ band: the $23.5 \mu \mathrm{m}$ band strength increases from 3 to 15 percent, while the $33.5 \mu \mathrm{m}$ band changes from 9 to 33 percent over continuum (for the highest disk mass). This is because the $33.5 \mu \mathrm{m}$ band probes regions further out in the disk, where the crystallinity difference between the two models becomes much more noticeable than in the inner regions. Indeed, Fig. 9 shows that the $R_{67}$ radius, which we define as the radius within which $2 / 3$ (or 67 percent) of the flux originates, differs more for the $33.5 \mu \mathrm{m}$ band than the $23.5 \mu \mathrm{m}$ band, when comparing the standard group I and group II models.

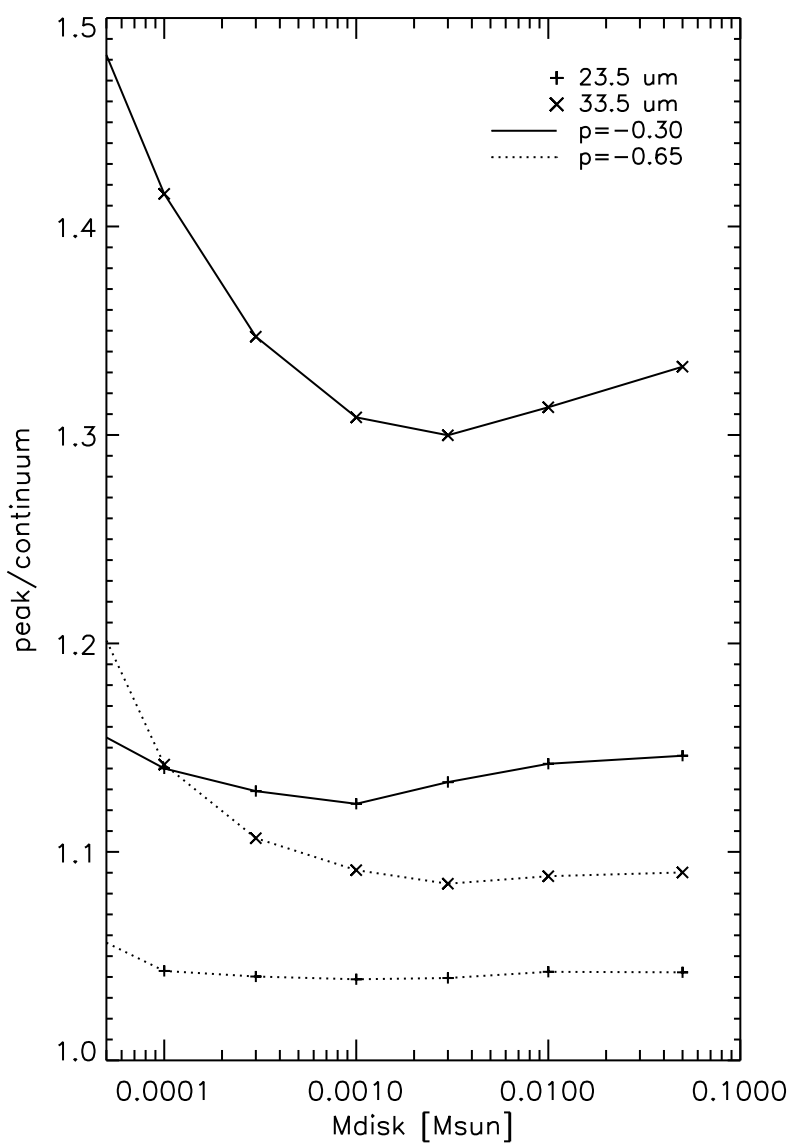

Fig. 8. Band strength of the 23.5 and $33.5 \mu \mathrm{m}$ bands for two scenario D models with $p=-0.65$ and $p=-0.30$. We use a range of disk masses, ranging from $10^{-4} M_{\odot}$ (the group II model mass) to $0.05 M_{\odot}$ (the group I model mass). All other model parameters are the same as for the standard model. Notice the initial decrease in band strength as dust mass increases.

Our models suggest that the $33.5 \mu \mathrm{m}$ band can be used as a measure of the radial mixing efficiency, assuming of course that the mechanism producing crystals at large distance from the star is indeed radial mixing. Care has to be taken however because the band strength depends not only on the radial distribution of crystals, but also on disk geometry.

The 23.5 and $33.5 \mu \mathrm{m}$ bands show a qualitatively different behaviour as a function of disk mass (and thus disk geometry). The $23.5 \mu \mathrm{m}$ band stays roughly constant with increasing disk mass, while the $33.5 \mu \mathrm{m}$ band strength initially decreases with increasing disk mass, followed by a slight increase again towards the highest disk masses used in our grid. Let us first discuss the behaviour of the $33.5 \mu \mathrm{m}$ band. The models with the lowest disk mass have strongly self-shadowed disks, and most of the contribution to the band strength comes from regions located relatively close to the star. These regions are more crystalline than the outer regions, and therefore the band strengths tend to be high for low dust mass, self-shadowed models. As the dust mass increases, more outer disk material contributes to the flux at $33.5 \mu \mathrm{m}$, with lower crystallinity. Therefore the band strength will decrease with increasing disk mass. The two models with the highest disk mass show a small increase in the $33.5 \mu \mathrm{m}$ band strength again. This can be attributed to the locus where the disk emerges from the shadow of the inner rim. This locus moves inwards for models with the highest dust masses, thus emphasising again regions with higher (inner disk) crystallinity. 


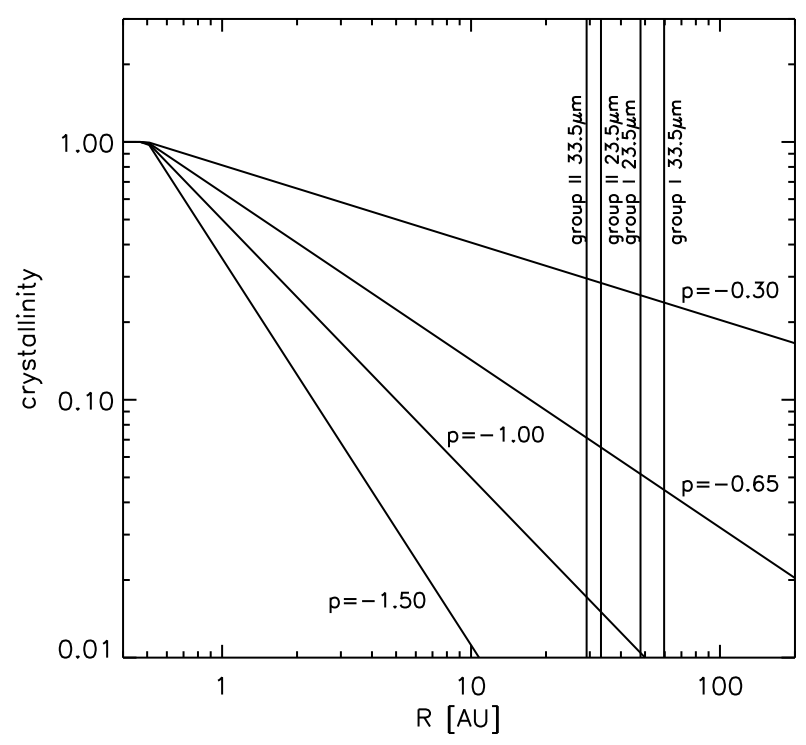

Fig. 9. For scenario D we show the radial dependence of crystallinity for four values of the parameter $p$, i.e. $p=-0.30,-0.65$ (the standard model value), -1.00 and -1.50 . Also indicated is the $R_{67}$ radius (defined as the radius within which $2 / 3$ of the total flux originates) for the two standard models, i.e. with $p=-0.65$, at a wavelength of 23.5 and $33.5 \mu \mathrm{m}$. These wavelengths coincide with prominent forsterite bands.

The $23.5 \mu \mathrm{m}$ band strength is much less dependent on disk geometry (mass). This is because the $23.5 \mu \mathrm{m}$ in our models is more dominated by the inner disk regions, and the (flaring) outer disk regions contribute less to the band strength. The degree of flaring is determined by the disk mass, but it affects less the structure of the inner disk regions. Therefore the $23.5 \mu \mathrm{m}$ band strength is less sensitive to disk mass. Figure 9 shows that group I and group II models have $R_{67}$ at $23.5 \mu \mathrm{m}$ that are more similar than the $R_{67}$ at $33.5 \mu \mathrm{m}$, and therefore the $23.5 \mu \mathrm{m}$ feature should be less sensitive to disk geometry than the $33.5 \mu \mathrm{m}$ band, as is seen in our model spectra.

\section{Conclusions}

The main results of this study can be summarised as follows:

1. The $5-40 \mu \mathrm{m}$ spectral shape is strongly affected by the mass in small grains. The group I spectra show higher flux levels than group II, reflecting the larger solid angle they subtend when viewed from the star. The spectral shape of group I and group II model spectra is rather similar in the wavelength range between 5 and $20-25 \mu \mathrm{m}$, but begins to differ substantially at longer wavelengths. At these longer wavelengths the presence (group I) or absence (group II) of a flaring outer disk becomes most noticeable.

2. In the studied wavelength range our models show clear evidence for the presence of crystalline silicates only when there is strong radial mixing, or other processes that increase the abundance of crystalline silicates at large distance from the star. Specifically, models that do not have crystalline silicates other than in the inner 1-2 AU show only very little evidence for crystalline silicates in the $10 \mu \mathrm{m}$ spectral region, and show no emission from this material at longer wavelengths.

3. The strength of the $10 \mu \mathrm{m}$ silicate bump (amorphous and crystalline) is affected by the amount of carbon dust assumed to be present in the models. However, in the $30 \mu \mathrm{m}$ wavelength range the carbon opacity is much less important. The $33.5 \mu \mathrm{m}$ forsterite band strength therefore is not significantly affected by the carbon dust abundance.

4. The $23.5 \mu \mathrm{m}$ and $33.5 \mu \mathrm{m}$ bands of forsterite probe different regions of the disk. The $23.5 \mu \mathrm{m}$ band is formed closer to the star, and its strength is not strongly affected by the disk geometry. However, the $33.5 \mu \mathrm{m}$ band has substantial contributions from regions between 20 and $50 \mathrm{AU}$. This is also the distance where the presence or absence of a flaring disk component is most noticeable. Therefore the $33.5 \mu \mathrm{m}$ band strength is a function of both the degree of crystallinity of the outer disk, and the disk geometry.

5. The mid-IR flux emerging from our models decreases with increasing grain size (using the same disk structure). This is because the dust opacity decreases with increasing grain size, causing the mid-IR emission to arise from deeper layers in the disk. The effect is most prominent for the group II model spectra.

Acknowledgements. J.M. acknowledges financial support from Network II of the Nederlandse Onderzoeksschool Voor Astronomy (NOVA) through project 10.10.2.12 and from the NWO Council for Physical Sciences through project 614.041.007.

\section{References}

Acke, B., \& van den Ancker, M. E. 2004, A\&A, 426, 151

Bockelée-Morvan, D., Gautier, D., Hersant, F., Huré, J.-M., \& Robert, F. 2002, A\&A, 384, 1107

Bohren, C. F., \& Huffmann, D. R. 1983, Absorption and scattering of light by small particles (New York: Wiley)

Bouwman, J., Meeus, G., de Koter, A., et al. 2001, A\&A, 375, 950

Calvet, N., Hartmann, L., \& Strom, S. E. 2000, Protostars and Planets IV, 377

Calvet, N., Muzerolle, J., Briceño, C., et al. 2004, AJ, 128, 1294

D’Alessio, P., Canto, J., Calvet, N., \& Lizano, S. 1998, ApJ, 500, 411

D’Alessio, P., Calvet, N., Hartmann, L., Franco-Hernández, R., \& Servín, H. 2006, ApJ, 638, 314

de Graauw, T., Haser, L. N., Beintema, D. A., et al. 1996, A\&A, 315, L49

Desch, S. J. 2007, ApJ, 671, 878

Draine, B. T., \& Lee, H. M. 1984, ApJ, 285, 89

Dullemond, C. P., \& Natta, A. 2003, A\&A, 408, 161

Dullemond, C. P., \& Dominik, C. 2004, A\&A, 417, 159

Dullemond, C. P., Apai, D., \& Walch, S. 2006, ApJ, 640, L67

Dullemond, C. P., Henning, T., Visser, R., et al. 2007, A\&A, 473, 457

Gail, H.-P. 2004, A\&A, 413, 571

Haisch, Jr., K. E., Lada, E. A., \& Lada, C. J. 2001, ApJ, 553, L153

Hellyer, B. 1970, MNRAS, 148, 383

Henning, T., Launhardt, R., Steinacker, J., \& Thamm, E. 1994, A\&A, 291, 546 Hillenbrand, L. A., Strom, S. E., Vrba, F. J., \& Keene, J. 1992, ApJ, 397, 613

Honda, M., Kataza, H., Okamoto, Y. K., et al. 2004, ApJ, 610, L49

Hony, S., Waters, L. B. F. M., \& Tielens, A. G. G. M. 2002, A\&A, 390, 533

Houck, J. R., Roellig, T. L., van Cleve, J., et al. 2004, ApJS, 154, 18

Isella, A., \& Natta, A. 2005, A\&A, 438, 899

Jäger, C., Molster, F. J., Dorschner, J., et al. 1998, A\&A, 339, 904

Kessler-Silacci, J., Augereau, J.-C., Dullemond, C. P., et al. 2006, ApJ, 639, 275 Laor, A., \& Draine, B. T. 1993, ApJ, 402, 441

Mathis, J. S., Rumpl, W., \& Nordsieck, K. H. 1977, ApJ, 217, 425

Meeus, G., Waters, L. B. F. M., Bouwman, J., et al. 2001, A\&A, 365, 476

Meijer, J., de Koter, A., Min, M., et al. 2007, A\&A, submitted

Meijer, J., Dominik, C., de Koter, A., et al. 2008, A\&A, 492, 451

Min, M., Hovenier, J. W., \& de Koter, A. 2003, A\&A, 404, 35

Min, M., Hovenier, J. W., \& de Koter, A. 2005, A\&A, 432, 909

Natta, A., Testi, L., \& Randich, S. 2006, A\&A, 452, 245

Preibisch, T., Ossenkopf, V., Yorke, H. W., \& Henning, T. 1993, A\&A, 279, 577

Servoin, J. L., \& Piriou, B. 1973, Phys. Stat. Sol. (b), 55, 677

van Boekel, R., Min, M., Leinert, C., et al. 2004, Nature, 432, 479

van Boekel, R., Min, M., Waters, L. B. F. M., et al. 2005, A\&A, 437, 189

Warren, J. L., Barret, R. A., Dodson, A. L., Watts, L. A., \& Zolensky, M. E.

1994, Cosmic Dust Catalog, Vol. 14, NASA Johnson Space Center, Houston Weidenschilling, S. J. 1977, Ap\&SS, 51, 153 